Local modification of GaAs nanowires induced by laser heating

This article has been downloaded from IOPscience. Please scroll down to see the full text article.

2011 Nanotechnology 22325701

(http://iopscience.iop.org/0957-4484/22/32/325701)

View the table of contents for this issue, or go to the journal homepage for more

Download details:

IP Address: 129.187.254.46

The article was downloaded on 14/07/2011 at $16: 33$

Please note that terms and conditions apply. 


\title{
Local modification of GaAs nanowires induced by laser heating
}

\author{
S Yazji ${ }^{1}$, I Zardo ${ }^{1}$, M Soini $^{1}$, P Postorino ${ }^{2}$, \\ A Fontcuberta i Morral ${ }^{1,3}$ and G Abstreiter ${ }^{1}$ \\ ${ }^{1}$ Walter Schottky Institut and Physik Department, Technische Universität München, \\ Am Coulombwall 4, D-85748 Garching, Germany \\ ${ }^{2}$ Dipartimento di Fisica, Universitá di Roma Sapienza, Piazzale Aldo Moro 5, I-00185 Roma, \\ Italy \\ ${ }^{3}$ Laboratoire des Matériaux Semiconducteurs, Institut des Matériaux, Ecole Polytechnique \\ Fédérale de Lausanne, CH-1015 Lausanne, Switzerland \\ E-mail: ilaria.zardo@wsi.tum.de
}

Received 21 March 2011, in final form 14 June 2011

Published 14 July 2011

Online at stacks.iop.org/Nano/22/325701

\begin{abstract}
GaAs nanowires were heated locally under ambient air conditions by a focused laser beam which led to oxidation and formation of crystalline arsenic on the nanowire surface. Atomic force microscopy, photoluminescence and Raman spectroscopy experiments were performed on the same single GaAs nanowires in order to correlate their structural and optical properties. We show that the local changes of the nanowires act as a barrier for thermal transport which is of interest for thermoelectric applications.
\end{abstract}

(Some figures in this article are in colour only in the electronic version)

\section{Introduction}

The interest in nanostructures has been steadily growing in recent years from a fundamental physics point of view as well as for potential technological applications. In this respect, nanowires are promising candidates for thermoelectric applications. The efficiency of a thermoelectric device is determined by the figure of merit ZT $=\sigma S^{2} T /\left(\kappa_{\mathrm{e}}+\kappa_{1}\right)$, where $\sigma$ is the electrical conductivity, $S$ the Seebeck coefficient, $\kappa_{\mathrm{e}}$ and $\kappa_{1}$ the electronic and lattice contributions to the thermal conductivity. This means that a material is suitable for thermoelectric applications if its electrical conductivity is high while the thermal conductivity $\kappa=\kappa_{\mathrm{e}}+\kappa_{\mathrm{l}}$ is low. Indeed, it has been predicted that nanowires should present an increased figure of merit with respect to the bulk material, because of increasing phonon boundary scattering and the appearance of quantized energy levels. Both phenomena arise from the confinement of the material at the nanoscale [1]. Though the electronic contribution to the thermal conductivity $\kappa_{\mathrm{e}}$ is connected to the electrical conductivity $\sigma$ by the WiedemannFranz law $\kappa_{\mathrm{e}}=\sigma L T$, with $L$ the Lorenz factor, a possibility for the improvement of thermoelectric performance is the tuning of the phonon thermal resistance in nanometer sized structures.
It has been shown that a local modification on a nanometer scale can be used to optimize the relevant functional properties of nanostructures $[2,3]$. Focused laser beams have been used for a long time for material processing for many different purposes [4]. Among these are laser induced crystallization, annealing, doping, alloying, oxidation and interdiffusion [5-9]. Laser induced processing enables high spatial resolution and position control.

In other respects the effects of laser heating while investigating nanostructures with spectroscopic techniques have always been a source of scientific debate, for both unambiguous evidence of phonon confinement and assignment of additional phonon modes [10-12].

In this work we investigate the effect of local laser heating on the structural, optical, and thermal properties of GaAs nanowires using a focused laser beam under ambient conditions. We demonstrate also that the local laser treatment leads to a strongly reduced thermal conductivity along the nanowires.

\section{Experimental details}

GaAs nanowires were grown by molecular beam epitaxy by the gallium assisted method, as described, for example, 
in [13]. The growth rate of the nanowires is given by the arsenic beam flux. The nanowires exhibit pure zinc-blende structure or wurtzite/zinc-blende heterostructures, depending on the growth conditions $[14,15]$. The investigated nanowires were grown at $\mathrm{As}_{4}$ pressures between $3.5 \times 10^{-6}$ and $8.8 \times$ $10^{-7}$ mbar, which results in GaAs nanowires with $100 \%$ zinc-blende structure and with $70 \% / 30 \%$ zinc-blende/wurtzite phase, respectively. The experimental results presented here were obtained on pure zinc-blende GaAs nanowires, albeit similar results were obtained also on nanowires with zincblende/wurtzite structure.

The nanowires were transferred onto a patterned substrate in order to identify single wires, which were then investigated by different techniques. The morphology and the optical properties of the nanowires were characterized by atomic force microscopy (AFM) and photoluminescence (PL) measurements, respectively, before and after laser heating. After laser heating, the morphologies of the nanowires were investigated also by scanning electron microscopy (SEM). Low temperature PL spectroscopy experiments on single nanowires were carried out by the use of a confocal microscope embedded in a $\mathrm{He}^{4}$ cryostat. Piezo-positioners allowed us to scan the sample with a spatial accuracy of few nanometers. A semiconductor laser diode with emission at $785 \mathrm{~nm}$ was used as excitation source and focused onto the sample with an objective with numerical aperture (NA) of 0.65 . The spot size of the laser in the confocal microscope was about $800 \mathrm{~nm}$. The luminescence was detected and analyzed by the combination of a grating spectrometer and a Peltier cooled Si charge coupled device. The investigation of the structural changes was performed by Raman spectroscopy. The $514.5 \mathrm{~nm}$ line of an $\mathrm{Ar}^{+}$laser was used for excitation and it was focused onto the nanowire with a $100 \times$ objective (NA $=0.95)$. The corresponding full width at half maximum (FWHM) of the Gaussian laser spot was measured to be $540 \pm 20 \mathrm{~nm}$. The scattered light was collected by an $X Y$ Raman Dilor triple spectrometer with a multichannel charge coupled device detector. The sample was positioned on an $X Y$ piezo-stage, which allowed scanning of the surface with a precision of about $10 \mathrm{~nm}$.

The laser heating at selected positions along the nanowires was performed by focusing the laser beam to a certain position at different powers. The laser beam used for this purpose provided the excitation light for the Raman spectroscopy experiments simultaneously. The local temperatures were obtained for different laser powers from the shifts of the phonon modes.

\section{Results}

Typical AFM and spatially resolved confocal PL measurements obtained from an as-grown GaAs nanowire are shown in figures 1(a) and (c), respectively. The diameter and its variation along the nanowire axis were determined by the height profile along the nanowire growth direction obtained from the AFM measurement. The nanowire exhibits a tapered shape, with a diameter of $97 \pm 2 \mathrm{~nm}$ at the bottom and $55 \pm 2 \mathrm{~nm}$ at the top, and a diameter of $60 \pm 2 \mathrm{~nm}$ in the middle.
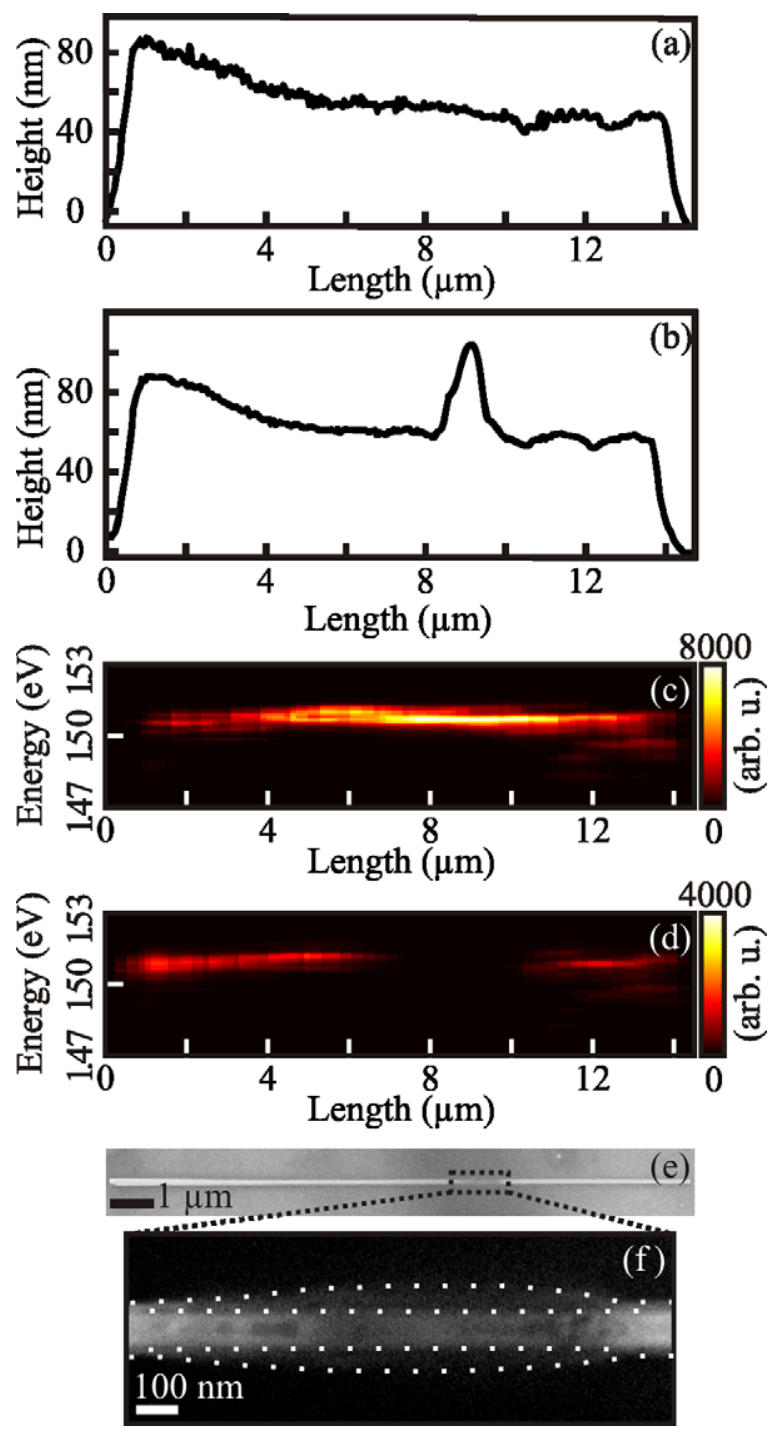

Figure 1. Cross section along the wire axis as determined by the AFM measurements before (a) and after (b) the laser induced heating. The diameter of the nanowire varies between 55 and $97 \mathrm{~nm}$. The position where the diameter is increased corresponds to the position where the laser heating was performed. Spatially resolved confocal PL measurements along the same nanowire before (c) and after (d) the laser induced heating. The intensity scale of the PL scan in (c) is double that in (d). (e) Top view scanning electron micrograph of the nanowire after laser heating. The detail of the exposed part of the nanowire is depicted in (f). The white dotted lines in (f) are a guide to the eyes.

We performed the laser induced local heating on selected positions along the nanowire length. The exposure time was kept constant to approximately 10 min for each power density. Several Raman spectra were collected during each exposure for shorter time intervals in order to watch the time evolution of the structural changes. Raman spectra as a function of the power density are shown in figure 2(a). At low power density $\left(140 \mathrm{~kW} \mathrm{~cm}^{-2}\right)$, the spectrum is composed exclusively of the transverse optical (TO) mode of GaAs at about $268 \mathrm{~cm}^{-1}$. With increasing power density (i.e. temperature), in addition to the down shift and broadening of the GaAs TO mode caused by heating, we observe the presence of a broad feature centered 

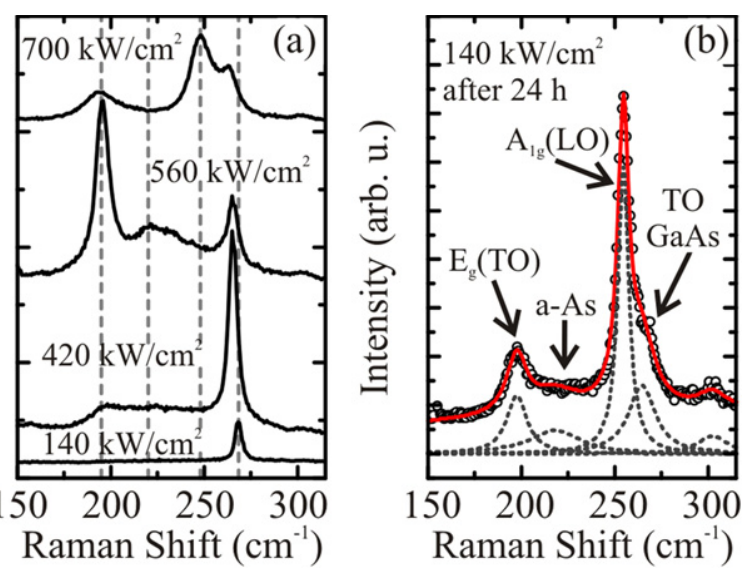

Figure 2. (a) Selection of Raman spectra collected by increasing the laser power on a single GaAs nanowire. The corresponding power densities are indicated. The polarization of the excitation was parallel to the nanowire axis. For the sake of clarity, the spectra are shifted vertically. The dashed lines indicate the positions of the TO mode of GaAs, the a-As peak, and the $\mathrm{E}_{\mathrm{g}}(\mathrm{TO})$ and $\mathrm{A}_{1 \mathrm{~g}}(\mathrm{LO})$ modes of c-As. (b) Raman spectrum collected at low power density, $24 \mathrm{~h}$ after the laser heating. The open circles are the experimental points, the dashed lines are the individual Lorentzian contributions, and the solid line is the combined fit curve.

at about $220 \mathrm{~cm}^{-1}$, followed by the formation of a sharper peak at $195 \mathrm{~cm}^{-1}$. The latter peak appears when the local temperature is increased by about $180 \mathrm{~K}$, as determined from the known temperature dependence of the GaAs TO phonon frequency [16]. With further increase of the power density, the appearance of a peak at $250 \mathrm{~cm}^{-1}$ is finally observed. These peaks can be attributed to the $\mathrm{E}_{\mathrm{g}}(\mathrm{TO})$ and $\mathrm{A}_{1 \mathrm{~g}}(\mathrm{LO})$ phonon modes of crystalline arsenic (c-As) $\left(198 \mathrm{~cm}^{-1}\right.$ and $257 \mathrm{~cm}^{-1}$, respectively, at room temperature), and to vibrational modes related to amorphous arsenic (a-As) [17, 18]. The downshift of these peaks compared to the expected values can also be attributed to the high temperature of our sample [19]. The appearance of the $\mathrm{E}_{\mathrm{g}}(\mathrm{TO})$ mode before the $\mathrm{A}_{1 \mathrm{~g}}(\mathrm{LO})$, shown also by the time evolution of the Raman spectra discussed in the following (figure 3), suggests an evolution and change in the crystal ordering of c-As.

The control spectrum in figure 2(b) was measured at low power density, $24 \mathrm{~h}$ after the nanowire was exposed to the focused laser beam. The spectrum is composed of four peaks located at 198, 220, 257 and $267 \mathrm{~cm}^{-1}$. The peak at $267 \mathrm{~cm}^{-1}$ is the TO mode of GaAs. The other three peaks correspond to crystalline and amorphous arsenic, demonstrating the irreversible structural changes of the nanowire.

In order to investigate the induced changes of the morphology and of the optical properties of the nanowire, we performed AFM, SEM, and PL measurements on the same nanowire after the treatment. The results are shown in figures 1(b) and (d)-(f). The AFM cross section of figure 1(b) reveals that the diameter of the nanowire is increased by about $50 \mathrm{~nm}$ at the annealed position. The SEM micrograph in figures 1(e) and (f) shows the increased diameter of the exposed part of the nanowire from about $90 \mathrm{~nm}$ to about $190 \mathrm{~nm}$, in agreement with the AFM results. The region of the nanowire exposed to laser heating exhibits a low contrast shell which

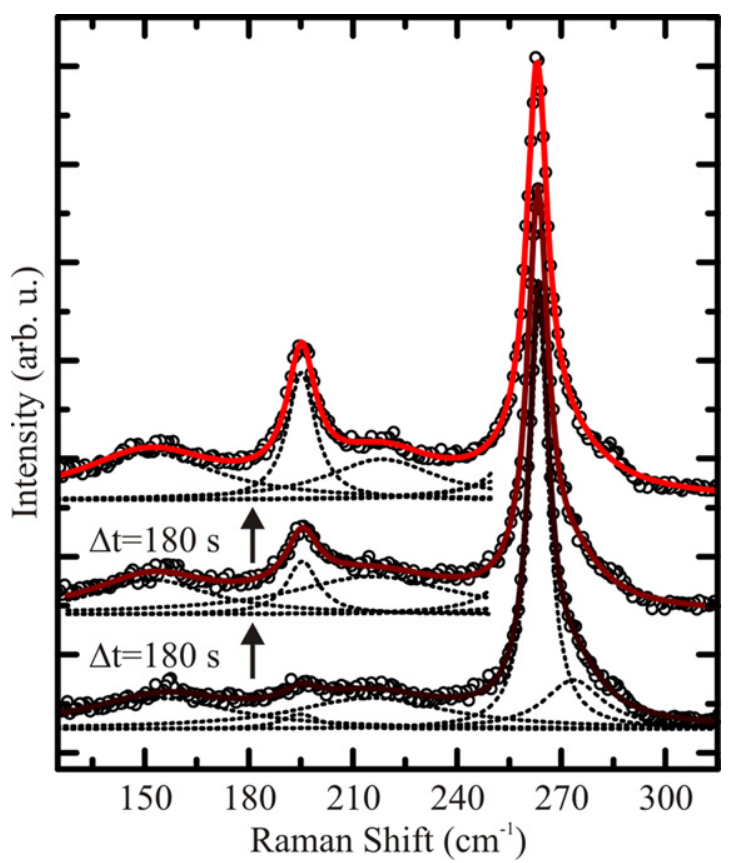

Figure 3. Time evolution of Raman spectra collected from a GaAs nanowire. The open circles are the experimental points, the dashed lines are the individual Lorentzian contributions, and the solid line is the combined fit curve. Each spectrum was collected for $180 \mathrm{~s}$ with an excitation power of $180 \mu \mathrm{W}$, corresponding to a power density of $79 \mathrm{~kW} \mathrm{~cm}^{-2}$. The polarization of the excitation was parallel to the nanowire axis.

is consistent with an oxide shell. The SEM micrograph does not show an indication of the presence of crystalline As, which is however clearly discernible in the Raman spectra. Interestingly, the core of the nanowire with a different contrast can be recognized and it is slightly reduced in diameter though uninterrupted. Both AFM and SEM measurements show that the diameter increase occurs isotropically. Furthermore, the PL signal disappears in this region of the wire (figure 1(d)). Similar results were found on all the investigated nanowires, with pure zinc-blende as well as zinc-blende/wurtzite structure, with high reproducibility. The formation of crystalline As due to heating of GaAs has already been observed and studied under several different experimental conditions [20-23]. It was proposed that the formation of crystalline As on GaAs films is mediated by the formation of arsenic oxide, which decomposes at the oxide/GaAs interface following the thermally induced interfacial solid-solid reaction $\mathrm{As}_{2} \mathrm{O}_{3}(\mathrm{~s})+$ $2 \mathrm{GaAs}(\mathrm{s}) \rightarrow \mathrm{Ga}_{2} \mathrm{O}_{3}(\mathrm{~s})+4 \mathrm{As}(\mathrm{s})$ [20]. In order to verify that the As formation was induced by the same mechanism, we exposed the nanowires to rapid thermal annealing under oxygen-free conditions for annealing temperatures up to $900 \mathrm{~K}$ and annealing time up to $5 \mathrm{~min}$. The Raman spectrum was measured subsequently using low power conditions. In this case, no formation of crystalline As was observed. In addition, Raman measurements were performed in vacuum $\left(10^{-5}\right.$ mbar) at similar high laser powers to those used in the experiments described above, without detecting the formation of crystalline As.

The threshold power at which the formation of crystalline As was observed under ambient conditions ranged from 360 
to $560 \mathrm{~kW} \mathrm{~cm}^{-2}$. The uncertainty in the determination of the threshold power is most probably related to the dependence of the absorbed power on the size, geometry and orientation of the nanowire $[24,25]$. However, it is possible to estimate the local temperature at which the formation of c-As is completed from the GaAs TO phonon frequency. For all the investigated nanowires, regardless of the crystal structure, the formation of c-As was completed when the local temperature was increased by about $200-300 \mathrm{~K}$. Again, only a temperature window in which the process occurs can be estimated, since only discrete power densities were used for the measurements. Furthermore, the reaction is a dynamic process with a temporal evolution, as is shown by the time evolution of the Raman spectra in figure 3 .

The spectra were collected from the middle of a suspended nanowire keeping the laser power constant at $180 \mu \mathrm{W}$ (corresponding to a power density of $79 \mathrm{~kW} \mathrm{~cm}^{-2}$ ) consecutively. It is worth noting here that in the case of suspended nanowires there is no heat diffusion from the nanowire to the substrate. Therefore, this leads to a higher local temperature for the same power density on the suspended nanowire compared to the case in which the nanowire is in contact with the substrate. The time evolution of the spectra is given by the emergence of the $\mathrm{E}_{\mathrm{g}}(\mathrm{TO})$ mode at $195 \mathrm{~cm}^{-1}$ and the two broad features at 152 and $220 \mathrm{~cm}^{-1}$, related to a-As, while no $\mathrm{A}_{1 \mathrm{~g}}(\mathrm{LO})$ mode is visible. The intensity of the modes related to the heating products increases with time. Interestingly, the intensity of the TO GaAs peak is nearly constant during the measurement, indicating that the structural changes are limited to a relatively thin shell around the nanowire. This holds as long as the oxide/As shell around the GaAs nanowire is thin enough and the GaAs core does not get thinned substantially by the material loss due to the solidsolid reaction, i.e. as long as the heating process is at its initial stage, like in the measurements of figure 3 .

These laser heating studies open up the possibility of modifying the structure and the properties of the nanowires locally. The oxide/As shell induced by local laser heating leads to additional phonon scattering and thus to a change of the thermal properties of the nanowires, like the thermal conductivity [25].

We investigated the qualitative modification of the thermal properties of the nanowires induced by the local laser treatment by a method based on the laser heating of freely suspended nanowires and the determination of the local temperature by Raman spectroscopy, as recently published [25]. The temperature profile obtained from an as-grown zinc-blende GaAs nanowire at a low laser power of $40 \mu \mathrm{W}$ (corresponding to a power density of $18 \mathrm{~kW} \mathrm{~cm}{ }^{-2}$ ) is shown by the open circles in figure 4 . The parabolic shape is an indication of the homogeneity of the system. The center part of the suspended nanowire was then exposed to laser irradiation for $540 \mathrm{~s}$ at a power of $150 \mu \mathrm{W}$, corresponding to a power density of $66 \mathrm{~kW} \mathrm{~cm}^{-2}$ and resulting in a rise in temperature to about $570 \mathrm{~K}$. Afterward, the temperature profile was measured with the low laser power as used for the original measurement (open squares). The temperature profile changed from parabolic to a linear behavior.

The local heat treatment at the center of the suspended nanowire has led to the creation of high thermal resistance

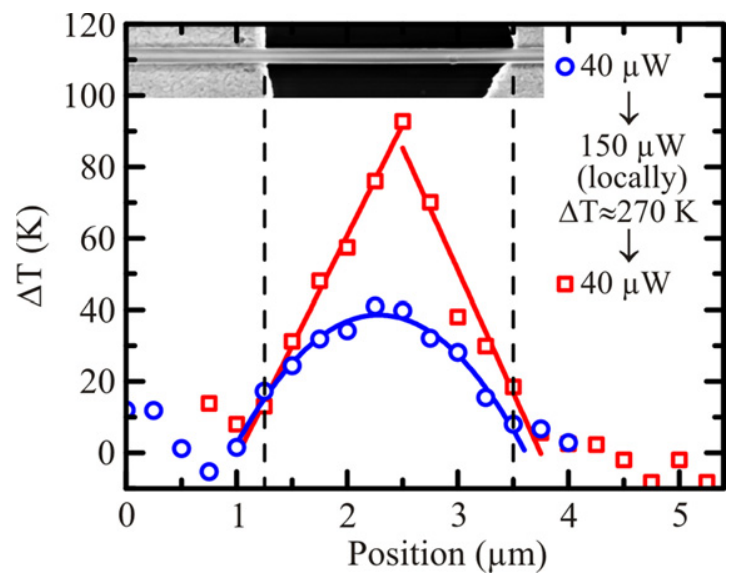

Figure 4. Temperature profiles obtained for homogeneous nanowires, before (open circles) and after (open squares) exposing the centers of the nanowires to laser heating. The symbols indicate experimental data points; the solid lines are parabolic and linear fits. The dashed lines indicate the positions of the gold pads. The inset shows an SEM micrograph of the corresponding freestanding nanowire. The scale bar of the micrograph is the same as the graph axis.

at the corresponding position. The thermal conductivity in this region can be assumed to be much lower compared to the thermal conductivity of the rest of the nanowire. The linear temperature profile can be understood by assuming heat transport only to the heat sinks on the left- or right-hand sides but not through the center part. This behavior can be understood quantitatively by calculating the ratio of the thermal conductivities obtained from the parabolic $\left(\kappa_{\mathrm{par}}\right)$ and linear temperature profiles $\left(\kappa_{\mathrm{lin}}\right)$. It is given by

$$
\frac{\kappa_{\mathrm{lin}}}{\kappa_{\mathrm{par}}}=\frac{P_{\mathrm{abs}} /(A|B|)}{P_{\mathrm{abs}} L /\left(4 \Delta T_{\max } A\right)}=\frac{4 \Delta T_{\max }}{L|B|}
$$

where $P_{\text {abs }}$ denotes the absorbed laser power, $B$ the slope of the linear curve, $A$ the nanowire cross section area, $L$ the length of the suspended part, and $\Delta T_{\max }$ the maximum heating in the middle of the parabolic curve. With the values $\Delta T_{\max }=$ $35 \mathrm{~K}, L=2.1 \mu \mathrm{m}$, and $|B|=68 \mathrm{~K} \mathrm{\mu m}^{-1}$ for the right-

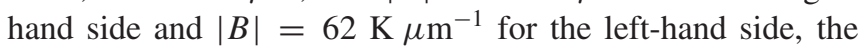
ratios of the conductivities are $\frac{\kappa_{\text {in }}}{\kappa_{\text {par }}}=1.0$ and $\frac{\kappa_{\text {in }}}{\kappa_{\text {par }}}=1.1$, respectively. This means that the thermal conductivity of the nanowire itself does not change and therefore we conclude that the structural changes leading to high thermal resistance are limited to a narrow region in the middle. Furthermore, this confirms that the oxide and crystalline As shell formation is an effect induced by the light-matter interaction rather than by the heating, in agreement with the work of Campbell et al [21]. Indeed, the process occurs and it is limited at the surface of the nanowire, while the temperature is reasonably constant across the nanowire. This means that the exposure to the laser catalyzes the solid-solid reaction.

\section{Conclusion}

In summary, we have presented a study of the effect of local laser induced modification of GaAs nanowires. The laser 
heating causes the formation of crystalline As, as proven by Raman scattering experiments and confirmed by the change in the morphology of the nanowire. The PL emission is completely suppressed in the annealed region. The structural changes are related for the first time to the thermal properties of the nanowires. It is shown that the formation of oxide and crystalline As leads to a local increase of the thermal resistance, as demonstrated by the determination of the thermal conductivity of GaAs nanowires by spatially resolved Raman spectroscopy. This opens possibilities to tailor and manipulate the thermal conductivity of nanowires and in this way to optimize their thermoelectric effects. Indeed, the geometry of the nanowire allows use of the change induced in the nanowire for thermoelectric purposes, since the core can still enable charge transport, while the local decrease of the thermal conductivity is provided by the formation of the oxide shell, which acts as a barrier for thermal transport. In addition, the laser induced oxide shell can be used for selective functionalization, after chemical removal of the c-As. In any case, this study gives a clear indication of the limits under which spectroscopic investigations on single nanowires can be performed under ambient air conditions in terms of laser power density.

\section{Acknowledgments}

We thank M Bichler, H Riedl, and N Hauke for excellent experimental help. This work was supported financially by the DFG via the excellence cluster Nanosystems Initiative Munich (NIM) and the SFB 631, the Institute for Advanced Study (IAS) of the TU München, and the Marie Curie Excellence Grant 'SENFED'.

\section{References}

[1] Hicks L D and Dresselhaus M S 1993 Phys. Rev. B 4716631

[2] Jung Y, Nam S W and Agarwal R 2011 Nano Lett. 11 1364-8
[3] Moriya R, Kobayashi H, Shibata K, Masubuchi S, Hirakawa K, Ishida S, Arakawa Y and Machida T 2010 Appl. Phys. Express 3035001

[4] Bäuerle D 2002 Appl. Surf. Sci. 186 1-6

[5] Kolb G, Salbert T and Abstreiter G 1991 J. Fresenius Anal. Chem. 341 166-70

[6] Chimmalgi A, Hwang D J and Grigoropoulos P 2005 Nano Lett. 5 1924-30

[7] Huber M, Deutschmann R A, Neumann R, Brunner K and Abstreiter G 2000 Appl. Surf. Sci. 168 204-7

[8] Baumgartner P, Brunner K, Abstreiter G, Böhm G, Tränkle G and Weimann G 1994 Appl. Phys. Lett. $64592-4$

[9] Brunner K, Abstreiter G, Walther M, Böhm G and Tränkle G 1992 Surf. Sci. 267 218-22

[10] Piscanec S, Cantoro M, Ferrari A C, Zapien J A, Lifshitz Y, Lee S T, Hofmann S and Robertson J 2003 Phys. Rev. B $68241312(\mathrm{R})$

[11] Adu K W, Gutiérrez H R, Kim U J and Eklund P C 2006 Phys. Rev. B 73155333

[12] Begum N, Piccin M, Jabeen F, Bais G, Rubini S, Martelli F and Bhatti A S 2008 J. Appl. Phys. 104104311

[13] Colombo C, Spirkoska D, Frimmer M, Abstreiter G and Fontcuberta i Morral A 2008 Phys. Rev. B 77155326

[14] Spirkoska D et al 2009 Phys. Rev. B 80245325

[15] Zardo I, Conesa-Boj S, Peiro F, Morante J R, Arbiol J, Uccelli E, Abstreiter G and Fontcuberta i Morral A 2009 Phys. Rev. B 80245324

[16] Besson J M, Itié J P, Polian A, Weill G, Mansot J L and Gonzales J 1991 Phys. Rev. B 449

[17] Farrow R L, Chang R K and Mroczkowski S 1977 Appl. Phys. Lett. $31768-70$

[18] Lannin J S 1977 Phys. Rev. B 15 3863-71

[19] Pizani P S, Campos C E M and Puech P 2000 Appl. Phys. Lett. 77 2924-5

[20] Schwartz G P, Schwartz B, Di Stefano D, Gualtieri G J and Griffiths J E 1978 Appl. Phys. Lett. 34205

[21] Campbell I H and Fauchet P M 1990 Appl. Phys. Lett. 5710

[22] Pizani P S and Campos C E M 1998 J. Appl. Phys. 846588

[23] Jain M, Datta G, Venkataraman P, Abbi S C and Jain K P 1989 Pramana J. Phys. 32641

[24] Cao L, White J S, Park J-S, Schuller J A, Clemens B M and Brongersma M L 2009 Nat. Mater. 8643

[25] Soini M, Zardo I, Uccelli E, Funk S, Koblmüller G, Fontcuberta i Morral A and Abstreiter G 2010 Appl. Phys. Lett. 97263107 